IZA DP No. 6877

An Alternative Explanation for the Variation in Reported Estimates of Risk Aversion

Denis Conniffe

Donal O'Neill

September 2012 


\title{
An Alternative Explanation for the Variation in Reported Estimates of Risk Aversion
}

\author{
Denis Conniffe \\ University College Dublin \\ Donal O'Neill \\ NUI Maynooth \\ and IZA

\section{Discussion Paper No. 6877 \\ September 2012} \\ IZA \\ P.O. Box 7240 \\ 53072 Bonn \\ Germany \\ Phone: +49-228-3894-0 \\ Fax: +49-228-3894-180 \\ E-mail: iza@iza.org
}

\begin{abstract}
Any opinions expressed here are those of the author(s) and not those of IZA. Research published in this series may include views on policy, but the institute itself takes no institutional policy positions. The IZA research network is committed to the IZA Guiding Principles of Research Integrity.

The Institute for the Study of Labor (IZA) in Bonn is a local and virtual international research center and a place of communication between science, politics and business. IZA is an independent nonprofit organization supported by Deutsche Post Foundation. The center is associated with the University of Bonn and offers a stimulating research environment through its international network, workshops and conferences, data service, project support, research visits and doctoral program. IZA engages in (i) original and internationally competitive research in all fields of labor economics, (ii) development of policy concepts, and (iii) dissemination of research results and concepts to the interested public.
\end{abstract}

IZA Discussion Papers often represent preliminary work and are circulated to encourage discussion. Citation of such a paper should account for its provisional character. A revised version may be available directly from the author. 


\section{ABSTRACT}

\section{An Alternative Explanation for the Variation in Reported Estimates of Risk Aversion ${ }^{1}$}

There is a large literature estimating Arrow-Pratt coefficients of absolute and relative risk aversion. A striking feature of this literature is the very wide variation in the reported estimates of the coefficients. While there are often legitimate reasons for these differences in the estimates, there is another source of variation that has not been considered to date. The Arrow-Pratt coefficients are properties of the utility functions, but a number of estimates are obtained by equating these to risk aversion measures defined in a mean-variance framework. This paper shows that while the legitimacy of the mean-variance approach may hold under general conditions the additional assumptions invoked when estimating the risk aversion parameter hold only in very restricted circumstances and that serious under or over estimation can easily arise as a result.

JEL Classification: C91, D81, G11

Keywords: variability in risk aversion estimation, mean-variance estimation, location scale

Corresponding author:

Donal O'Neill

Economics Department

NUI Maynooth

Maynooth, Co. Kildare

Ireland

E-mail: donal.oneill@nuim.ie

\footnotetext{
${ }^{1}$ The substantive analysis in this paper was carried out by Denis Conniffe before he passed away in January 2011. He was a remarkable man and a wonderful colleague and will be sadly missed by all those who knew him. At Denis's request, Donal O'Neill agreed to complete this paper and prepare it for submission. We are grateful to Tom Flavin and an anonymous referee for comments on an earlier draft of this paper.
} 


\section{Introduction}

There exists a large literature in which researchers seek to estimate the ArrowPratt coefficients of absolute and relative risk aversion. While a number of different approaches have been used two techniques have proved particularly popular. Experimental and survey methods often ask participants to price a hypothetical lottery and infer risk-aversion from the answers (for example Barsky et al 1997, Hartog et al 2002, Guiso and Paiella 2008). This approach has been evaluated recently by Donkers et al (2001) and Ding et al (2010). A second popular approach uses a mean variance framework and obtains measures of risk aversion by equating the Arrow Pratt measures to risk aversion measures defined in a mean-variance setting. Examples of this latter approach include Friend and Blume (1975); Frankel (1985), Engel and Rodrigues (1989), Giovanni and Jorion (1989), Thomas and Wickens (1993); Clare, O’Brien, Thomas and Wickens (1998), and Flavin (2006).

A striking feature of this literature is the large variation in the reported estimates of the risk-aversion coefficients. ${ }^{2}$ Meyer and Meyer (2005) put forward one possible explanation for this variation, noting how the magnitudes of relative risk aversion can depend greatly on the chosen argument of the utility function. In this paper we provide another possible explanation. Focusing on the studies that employ the mean-variance approach to estimate risk-aversion we show how failure of a key requirement in these models can easily lead to serious under or over estimation of the risk-aversion parameters. The key insight focuses on the relationship between measures of riskaversion based on the curvature of the Bernoulli utility function and measures of risk defined in mean-variance space. As is well known the Arrow-Pratt coefficients are based on the curvature properties of the utility function and are typically functions of income. Meyer (1987) describes a correspondence between the rates of change of risk aversion measures based on the utility function and alternatives defined in mean-variance space. However, he did not examine the validity of the key relationship required for the methodologies used in the above studies. In this paper we show that the legitimacy of this key equation holds only in very restricted circumstances and that different specifications of preferences or of the distributions governing uncertainty can easily generate significant

\footnotetext{
2 The estimated coefficient of relative risk aversion ranges from -142 to +11 in the studies cited above.
} 
variation in the estimated measures of risk-aversion. We show that the implications of our analysis are consistent with previous Monte Carlo evidence provided by Kocherlakota (1990) and discuss how our analysis may help understand these simulation results.

\section{Arrow-Pratt Measures of Risk aversion and the mean-variance framework}

Decision making involving a random variable $y$ is frequently based on maximizing expected utility

$$
E\{U(y)\}=V(\boldsymbol{\theta}),
$$

where $U(y)$ is some concave utility function and $\boldsymbol{\theta}$ is the vector of parameters of the distribution of the random variable $y$, e.g. final period wealth. Sometimes $\boldsymbol{\theta}$ has only two components and can be re-expressed in terms of $\mu_{y}$ and $\sigma_{y}$, the mean and standard deviation of $y$. Then

$$
E\{U(y)\}=V\left(\sigma_{y}, \mu_{y}\right)
$$

The natural measure of risk aversion when considering $V\left(\sigma_{y}, \mu_{y}\right)$ is the slope of the indifference curve; in mean-variance space these indifference curves consist of $\left(\sigma_{y}, \mu_{y}\right)$ combinations that are equally preferred. ${ }^{3}$ This slope $S=\frac{d \mu_{y}}{d \sigma_{y}}=-\left\{\frac{\partial V}{\partial \sigma_{y}}\right\} /\left\{\frac{\partial V}{\partial \mu_{y}}\right\}$ gives the increase in expected return required to compensate for a marginal increase in risk as measured by $\sigma_{y}$. For optimal solutions the derivatives of $V\left(\sigma_{y}, \mu_{y}\right)$ with respect to $\mu_{y}$ and $\sigma_{y}$ must be positive and negative respectively and the indifference curves associated with $V\left(\sigma_{y}, \mu_{y}\right)$ must be convex.

\footnotetext{
${ }^{3}$ See for example Hawawini (1978).
} 
However, risk aversion is usually introduced and discussed in terms of properties of $U($.$) . The Arrow-Pratt coefficients of absolute and relative risk aversion are functions$ over $y$ defined as

$$
R_{A}(y)=-\frac{U^{\prime \prime}(y)}{U^{\prime}(y)} \quad \text { and } \quad R_{R}(y)=y R_{A}(y) \text {, }
$$

with $R_{R}$ having the virtue of being dimensionless. Estimates of the Arrow-Pratt measure are typically reported at particular values of $y$ such as $y=\mu_{y}$.

There is a large literature estimating the coefficients of absolute and relative risk aversion, often relying on the assumed equality

$$
\frac{1}{\sigma_{y}} S=R_{A}\left(\mu_{y}\right)
$$

and consequently

$$
\frac{\mu_{y}}{\sigma_{y}} S=R_{R}\left(\mu_{y}\right)
$$

Examples of studies that use this equality include Friend and Blume (1975), Frankel (1985), Engel and Rodrigues (1989), Giovanni and Jorion (1989), Thomas and Wickens (1993), Clare, O’Brien, Thomas and Wickens (1998) and Flavin (2006). Of course most of these authors had other objectives besides estimating risk aversion measures and the argument $y$ in the utility function $U(y)$ differed from study to study. Meyer and Meyer (2005) have illustrated how the chosen argument can significantly affect estimates of risk aversion. However, we show that this is not the only factor that could potentially be responsible for the divergence in reported estimates. In this paper we argue that the use of (1) is another and perhaps more important source of varying estimates.

Deriving a numerical estimate of $R_{R}$ requires observation of allocation under risk. In the much used simple case of an investor allocating between a risky asset with a single time period return $x$, distributed with mean $\mu$ and variance $\sigma^{2}$ per unit and a riskless 
asset with (for convenience) return unity, optimal choice of the proportion $p$ of available wealth allocated to the risky asset follows from maximization with respect to $p$ of

$$
E\left[U\left\{W_{0}(1-p+p x)\right\}\right]=E[U(y)]=V\left(\sigma_{y}, \mu_{y}\right),
$$

where $\mu_{y}=W_{0}(1-p+p \mu)$ and $\sigma_{y}=W_{0} p \sigma$. In this simple model, with a single risky and riskless asset, final wealth depends linearly on the random variable $x$. It follows therefore that all final wealth random variables follow a location-scale distribution, no matter how $x$ is distributed. Thus the expected utility maximizing problem can be characterized in a mean-variance framework no matter what utility function is specified. Although written here in terms of final period wealth, in practice $y$ can refer either to the uncertain level of final wealth or to uncertain level of returns $(x)$ on the assets generating this wealth and one can easily translate between mean-variance preferences over assets to mean-variance preferences over returns.

Maximising expected return with respect to $p$ gives

$$
\frac{\partial V}{\partial \mu_{y}}(\mu-1) W_{0}+\frac{\partial V}{\partial \sigma_{y}} \sigma_{y} W_{0}=0
$$

or

$$
\mu-1=S\left(\sigma_{y}, \mu_{y}\right) \sigma_{y} .
$$

If (1) holds then $S\left(\sigma_{y}, \mu_{y}\right)=R_{A}\left(\mu_{y}\right) \sigma_{y}$ giving the familiar

$$
\mu-1=p R_{R} \sigma_{y}^{2}
$$

from which $R_{R}$ can be deduced from observed returns and their variance and the portfolio weights $p$.

Since the Arrow-Pratt measure typically varies over $y$ the approximation in (2) is at a particular point, $y=\mu_{y}$, in expected-utility space. Correspondingly the value of $\sigma_{y}$ 
that appears in (2) must be the value of $\sigma_{y}$ that corresponds to $\mu_{y}$ at the optimally selected portfolio. In most empirical estimation these returns and variances are estimated using elaborate analyses such as multivariate GARCH regression of returns on variances over multiple time periods (with $\mu, \sigma$ and $p$ varying over time).

The equality implied in (1) is exactly true in two well-known situations. The first involves a negative exponential utility function, where $U(y)=1-e^{-\lambda y}$, combined with a normal distributions for returns. In this case

$$
E\{U(y)\}=V\left(\sigma_{y}, \mu_{y}\right)=1-e^{\left(-\lambda \mu_{y}+\lambda^{2} \sigma_{y}^{2} / 2\right)},
$$

from which it easily follows $S / \sigma=\lambda$ and, of course, $R_{A}(y)=\lambda$. The second situation specifies an exact quadratic utility function, where $U(y)=a y-b y^{2}, y<a / 2 b$. In this case $V(\sigma, \mu)=a \mu-b \mu^{2}-b \sigma^{2}$ and the equality in (1) is easily verified.

While most accept that the increasing risk aversion implied by quadratic utility is counterintuitive it is true that any concave function can be adequately approximated over a short range by a quadratic. If points $\mu$ and $\alpha$ are sufficiently close, the power series

$$
U(y) \approx U(\alpha)+(y-\alpha) U^{\prime}(\alpha)+(y-\alpha)^{2} U^{\prime \prime}(\alpha) / 2+(y-\alpha)^{3} U^{\prime \prime \prime}(\alpha) / 3 !+\ldots
$$

can perhaps be adequately approximated by its first few terms. However, if we take $x$ to be a realisation of a random variable from a distribution with mean $\mu$ and variance $\sigma^{2}$ the possible 'distance' of $E\{U(y)\}$ from the first few terms of

$$
U(\alpha)+\left(\mu_{y}-\alpha\right) U^{\prime}(\alpha)+E(y-\alpha)^{2} U^{\prime \prime}(\alpha) / 2+E(y-\alpha)^{3} U^{\prime \prime \prime}(\alpha) / 3 !+\ldots .
$$

depends on the characteristics of the distribution, including $\mu_{y}, \sigma^{2}$ and higher moments. Samuelson (1970) was the first to specify rigorously what is required to reproduce (2) by quadratic approximation. 


$$
\begin{gathered}
E[U\{W(1-p+p x))\}]=U(W)+E(W p(x-1)) U^{\prime}(W)+W^{2} p^{2} E\left[(x-1)^{2}\right] U^{\prime \prime}(w) / 2+\ldots \\
=U(W)+W p(\mu-1) U^{\prime}(W)+W^{2} p^{2}\left[\left(\sigma^{2}+(\mu-1)^{2}\right] U^{\prime \prime}(w) / 2+\ldots\right.
\end{gathered}
$$

Then setting the derivative with respect to $\mathrm{p}$ to zero

$$
0=W(\mu-1) U^{\prime}(W)+W^{2} p\left[\left(\sigma^{2}+(\mu-1)^{2}\right] U^{\prime \prime}(w)+. .\right.
$$

gives (2) provided terms in powers of $\sigma^{2}$ higher than 2, $(\mu-1)^{2}$ and its higher powers are negligible. This requires not only that $\sigma$ be small, but that $\mu=1+O\left(\sigma^{2}\right)$. Then $(\mu-1)^{2}$ is $O\left(\sigma^{4}\right)$ and negligible. Samuelson called this situation of very limited riskiness and expected returns a 'compact' distribution and the restrictions on its applicability are obvious.

\section{Correspondence, but not equality, of Risk-Aversion Measures.}

Samuelson (1970) was motivated by a wish to defend mean-variance analysis and the optimality of diversification against the criticism by Feldstein (1969). His conclusion that mean-variance analysis could stand, at least for his 'compact' distribution as well as for normality, was actually pessimistic. Meyer (1987) and Sinn (1989) showed that equivalence of expected utility maximisation and mean-variance analysis could be extended from the normal distribution to the location-scale family of distributions, which are classes of distributions defined by having distribution functions of the form

$$
P(Y \leq y)=F\left(\frac{y-\beta}{\alpha}\right),
$$

where $\beta$ is the location and $\alpha$ the scale parameter. For the normal distribution these are the mean and standard deviation, but that need not be true of other members of the family. Meyer (1987) also described a correspondence between the rates of change of 
$R_{A}$ and $S$ showing that if $R_{A}{ }^{\prime}(y)$ is positive for all $y, S$ increases with $\mu$, if it is zero $S$ is constant in $\mu$ and if it is negative $S$ decreases with $\mu$. However he did not assert that (1) holds. Boyle and Conniffe (2008) extended the compatibility of expected utility maximisation and mean-variance analysis to distributions transformable to location-scale form by a concave transformation, at least for utility functions that remain concave under the same transformation. They found an analogous, although not as clear cut,

correspondence between the rates of change of $R_{A}$ and $S$ but again there was no implication that (1) would hold.

So while mean-variance analysis and its implications for diversification are actually more widely applicable than was initially appreciated, there is no guarantee that equality (1) carries over beyond normality or the 'compact' distribution case. That does not seem to have permeated to the literature estimating Arrow-Pratt risk aversion measures, leading to perhaps seriously inaccurate estimates.

\section{Retaining negative exponential utility, but varying the distribution from normality}

In this section we consider two alternative non-normal specifications for uncertainty in returns. Both of these alternatives is a two parameter distribution but they depart from normality in rather different ways.

\section{Location-scale with heavier tails}

The logistic distribution is very similar to the normal over most of its range, being of location scale form and symmetric about its mean, but with somewhat fatter tails. The occurrence of fat tailed distributions is frequently reported for financial data on returns and indeed distributions such as the ' $\mathrm{t}$ ' distribution with few degrees of freedom have been advocated as appropriate. These have much fatter tails than a logistic so the latter is perhaps a rather conservative choice.

The logistic distribution function is

$$
F(y)=\frac{1}{1+e^{-(y-\alpha) / \beta}} \quad-\infty<y<\infty
$$


with density

$$
f(y)=\frac{1}{\beta} \frac{e^{-(y-\alpha) / \beta}}{\left(1+e^{-(y-\alpha) / \beta}\right)^{2}} .
$$

The mean and standard deviation are $\alpha$ and $\pi \beta / \sqrt{3}$ respectively. So $\beta=\sqrt{3} \sigma / \pi$.

$$
\begin{gathered}
E\{U(y)\}=V(\sigma, \mu)=E\{1-\exp (-\lambda y)\}, \\
=1-\int_{-\infty}^{\infty} \exp (-\lambda y) f(y) d y
\end{gathered}
$$

Recognizing the second term in this expression as the moment generating function of the logistic distribution evaluated at $-\lambda$ we can rewrite this as

$$
V(\sigma, \mu)=1-e^{-\alpha \lambda} \Gamma(1+\beta \lambda) \Gamma(1-\beta \lambda), \quad \beta \lambda<1,
$$

Using the properties of the Gamma function this can be rewritten as

$$
\begin{gathered}
V(\sigma, \mu)=1-e^{-\alpha \lambda} \beta \lambda \Gamma(\beta \lambda) \Gamma(1-\beta \lambda), \\
=1-e^{-\alpha \lambda} \beta \lambda \frac{\pi}{\operatorname{Sin}(\beta \lambda \pi)} .
\end{gathered}
$$

Expressing $\alpha$ and $\beta$ in terms of $\mu$ and $\sigma$ gives

$$
V(\sigma, \mu)==1-e^{-\lambda \mu} \sqrt{3} \sigma \lambda \frac{1}{\operatorname{Sin}(\sqrt{3} \sigma \lambda)} .
$$

Note that $\operatorname{Sin}(\beta \lambda \pi)=\operatorname{Sin}(\sqrt{3 \sigma} \lambda)$ is positive. Taking derivatives 


$$
\frac{\partial V}{\partial \mu}=\lambda e^{-\lambda \mu} \sqrt{3} \sigma \lambda \frac{1}{\operatorname{Sin}(\sqrt{3} \sigma \lambda)}
$$

And

$$
\frac{\partial V}{\partial \sigma}=-\lambda e^{-\lambda \mu} \sqrt{3} \frac{1}{\operatorname{Sin}(\sqrt{3} \sigma \lambda)}\{1-\sqrt{3} \sigma \lambda \operatorname{Cotan}(\sqrt{3} \sigma \lambda)\}
$$

As expected the derivative with respect to $\mu$ is positive and the derivative with respect to $\sigma$ is negative.

$$
S=\frac{d \mu}{d \sigma}=-\left\{\frac{\partial V}{\partial \sigma}\right\} /\left\{\frac{\partial V}{\partial \mu}\right\}=\frac{1}{\sigma \lambda}\{1-\sqrt{3} \sigma \lambda \operatorname{Cotan}(\sqrt{3} \sigma \lambda)\}
$$

$S$ is not a function of $\mu$ and so, for fixed $\sigma$, risk aversion in the $(\mu, \sigma)$ metric does not change with $\mu$, which is of course consistent with the CARA and IRRA properties of $U(x)=A-\exp (-\lambda x)$ and really just a special cases of Meyer's results for location-scale distributions. By employing the accurate approximation (for example, Abramowitz and Stegun, 1972, p. 76) ; $x \operatorname{Cotan}(x) \approx 1-.333 x^{2}-.024 x^{4}$ equation (4) simplifies to

$$
S \approx \frac{\sigma^{2} \lambda^{2}\left\{1+.216 \sigma^{2} \lambda^{2}\right\}}{\sigma \lambda}=\sigma \lambda\left\{1+.216 \sigma^{2} \lambda^{2}\right\}
$$

Evidently $\frac{d S}{d \sigma}>0$ so that indifference curves are convex which, again was implied by Meyer's results.

However $\frac{1}{\sigma} S \neq \lambda$ and employing (1) will overestimate $R_{A}$ and consequently $R_{R}$ - Another interpretation is that risk aversion as measured in a $(\mu, \sigma)$ metric is larger than measures based on $U(x)$, which seems quite plausible given the heavier tails of the logistic distribution. Regarding the magnitude of overestimation, since $\sigma \lambda$ would be the 
slope of an indifference curve for CARA utility with normal returns, $\sigma^{2} \lambda^{2}$ cannot be considered negligible except for low $\sigma$. Obviously for a slope of unity the overestimation would be $22 \%$. We can also use the fact that value of $\sigma^{2}$ used in (1) is that associated with the optimal portfolio and the value of $\mu_{y}$ used to evaluate the Arrow-Pratt measure to consider the range of likely biases. Rotthoff (2011) reports a mean return and standard deviation on the NASDAQ stock exchange of $2.5 \%$ and $1.6 \%$ respectively over the period 1986-2009. To work out the bias we need a value for $\lambda$, the coefficient of absolute risk-aversion. Meyer and Meyer (2005) report a range of adjusted estimates for the coefficient of relative risk-aversion, with typical estimates ranging from ranging from 2 to 7 . Using the relationship between relative and absolute risk-aversion and our mean return we can back out the corresponding range for $\lambda$ needed for our calculations. This measure can then be combined with our estimate for the variance in returns to gauge the size of the bias using equation (5). These calculations show a bias ranging from 3.3\% when the coefficient of relative risk-aversion equals 2, rising to $41 \%$ when the coefficient of relative risk-aversion equals $7 .^{4}$ Bearing in mind that the logistic is only mildly fat tailed (roughly equivalent to a 't' distribution with 7 degrees of freedom), in practice serious overestimation is likely.

\section{Location-scale but skew}

The Gumbel distribution is another within the location-scale family and its density is

$$
f(y)=\frac{1}{\beta} e^{-\left(\frac{y-\alpha}{\beta}\right)} \exp \left(-e^{-\left(\frac{y-\alpha}{\beta}\right)}\right),
$$

However, unlike the normal and logistic distributions it is positively skew. Positively skew distributions are also sometimes reported in the literature on financial returns data. The mean and standard deviation are $\alpha+\gamma \beta$ and $\pi \beta / \sqrt{6}$ respectively, where $\gamma=.5772157 \ldots$ is Euler's constant.

\footnotetext{
${ }^{4}$ These estimates are based on a share of risky assets, $p$, equal to .3 (see for example Jianakoplos (2002) for the United States and Gusio and Paiella (2008) for Italy).
} 
Expected utility is

$$
V(\sigma, \mu)=E\{U(y)\}=E\{1-\exp (-\lambda y)\}=1-\int_{-\infty}^{\infty} \exp (-\lambda y) f(y) d y
$$

Again recognizing the second term as the moment generating function we can rewrite this as

$$
V(\sigma, \mu)=1-e^{-\alpha \lambda} \Gamma(1+\beta \lambda)=1-e^{(-\mu \lambda+\sqrt{6} \gamma \sigma \lambda / \pi)} \Gamma(1+\sqrt{6} \sigma \lambda / \pi) .
$$

Then

$$
\frac{\partial V}{\partial \mu}=\lambda e^{(-\mu \lambda+\sqrt{6} \gamma \sigma \lambda / \pi)} \Gamma(1+\sqrt{6} \sigma \lambda / \pi)
$$

and

$$
\frac{\partial V}{\partial \sigma}=-\lambda \frac{\sqrt{6}}{\pi} e^{(-\mu \lambda+\sqrt{6} \gamma \sigma \lambda / \pi)} \Gamma(1+\sqrt{6} \sigma \lambda / \pi)[\gamma+\Psi(1+\sqrt{6} \sigma \lambda / \pi)],
$$

where $\Psi$ denotes the Digamma function. The derivatives with respect to $\mu$ and $\sigma$ are positive and negative respectively and

$$
S=\frac{d \mu}{d \sigma}=-\left\{\frac{\partial V}{\partial \sigma}\right\} /\left\{\frac{\partial V}{\partial \mu}\right\}=\frac{\sqrt{6}}{\pi}\{\gamma+\Psi(1+\sqrt{6} \sigma \lambda / \pi)\} .
$$

Again, as implied by the location-scale property of the distribution, $S$ is not a function of $\mu$, matching the CARA property of negative exponential utility. But again $\frac{1}{\sigma} S \neq \lambda$.

For $|z|<1$ (6) can be rewritten by employing the series expansion for the Digamma function $\Psi(1+z)=-\gamma+\sum_{n=2}^{\infty}(-1)^{n} \zeta(n) z^{n-1}$ given, for example, in Abramowitz 
and Stegun, (1972, p. 259), where $\varsigma(n)$ is the Riemann-Zeta function. In particular, $\varsigma(2)=\pi^{2} / 6$. Then for $\sigma \lambda<1.3$ (6) becomes the more easily interpreted

$$
S=\sigma \lambda\left\{1+\sum_{n=3}^{\infty}\left(-\frac{\sqrt{6}}{\pi}\right)^{n} \zeta(n)(\sigma \lambda)^{n-2}\right\}
$$

The infinite sum within the brackets is negative and $S / \sigma$ could substantially underestimate $\lambda$ especially for large $\sigma$. In fact, using Abramowitz and Stegun tables, with $\sigma \lambda=.5$ (6) gives .4 and with $\sigma \lambda=1$ it gives .66. What is occurring is risk aversion as measured in a $(\mu, \sigma)$ metric is smaller than that measured from $U(y)$, which is plausible with a positively skew distribution.

\section{Changing the utility function}

By far the most popular alternatives to the negative exponential, or CARA, utility function are the power and log functions, which display constant relative risk aversion (CRRA). But their expected utilities for distributions with support from $-\infty<y<\infty$ (such as the normal, logistic and Gumbel) do not exist. The problem is $-\gamma y^{-\gamma}$ is imaginary for negative $y$ and non- integer $\gamma$ and complicated redefinition of the utility function is required, which upsets the risk aversion properties predicted for expected utilities from location-scale distributions. However, power and log utilities are most commonly associated with the lognormal distribution $0 \leq y<\infty$. Then, as shown in Boyle and Conniffe (2008), for the power function we get

$$
S=\frac{(1+\gamma) \mu \sigma}{\mu^{2}+(2-\gamma) \sigma^{2}}, \quad \gamma>-1
$$

where $\mu$ and $\sigma^{2}$ are the mean and variance of $\mathrm{x}$, not of $\log \mathrm{x}$. 


$$
\frac{\partial S}{\partial \mu}=S \frac{(2+\gamma) \sigma^{2}-\mu^{2}}{\mu\left(\mu^{2}+(2+\gamma) \sigma^{2}\right)}
$$

and so risk aversion decreases with $\mu$, provided $\mu^{2}>(2+\gamma) \sigma^{2}$. When untrue the indifference curve ceases to be convex. We know $R_{A}(\mu)=\frac{(1+\gamma)}{\mu}$ and $R_{R}(\mu)=(1+\gamma)$ But $S / \sigma \neq(1+\gamma) / \mu$ nor is $\mu S / \sigma$ constant. Instead

$$
\frac{\mu}{\sigma} S=\frac{(1+\gamma)}{1+(2+\gamma) \sigma^{2} / \mu^{2}}
$$

which can obviously substantially underestimate $(1+\gamma)$. The reason is again the positive skewness of the lognormal which reduces risk aversion in a $(\mu, \sigma)$ metric relative to that deduced from $U(x)$. In this context it is interesting to revisit the findings of Kocherlakota (1990). Using a Monte-Carlo approach in which agents have CRRA preferences he finds that the approach of Friend and Blume (1975) leads one to substantially underestimate of the coefficient of risk-aversion. ${ }^{5}$ Kocherlakota suggests that overestimation of the variance of returns may in part explain this result. However, given his assumption of CRRA and the positive skewness evident is his generated returns (see Table 1 page 291), the deviation of $\mu S / \sigma$ from $R_{R}(\mu)$ which we have highlighted offers another, potentially more obvious, explanation for these findings.

\section{Concluding Remarks}

The 'standard' conversion formula for deducing $R_{A}(\mu)$ or $R_{R}(\mu)$ from a mean-variance framework does not work except in a limited range of circumstances. Arguably, a risk aversion measure in a $(\mu, \sigma)$ metric is a more meaningful concept than $R_{A}(\mu)$ or $R_{R}(\mu)$. An investor or other decision maker will take into account not only their own 'risk

\footnotetext{
${ }^{5}$ The average estimate across his samples using Friend and Blume's approach was 1.8, while the true calibrated value was 13.7.
} 
tolerance' as deduced from their utility function for wealth, but also the distribution of outcomes they perceive themselves facing. This is by no means an original idea; for example, it is mentioned in passing in Friend and Blume (1975). However, the literature has been heavily weighted towards obtaining estimates of $R_{A}$ or $R_{R}$ and, as mentioned in the Introduction, the variation in reported estimates has been very large. There may be legitimate reasons for differences in estimates, but this paper has shown that either under or over estimation could arise from illegitimate application of the 'standard' conversion formula. It has also shown that the degree of under or over estimation can easily be very substantial.

\section{References}

Abramowitz, M. and I. Stegun (1972) Handbook of Mathematical Functions with Formulas, Graphs, and Mathematical Tables New York Dover publications.

Barsky, R. B., T. F. Juster, M. S. Kimball, and M. D. Shapiro (1997), Preference Parameters and Individual Heterogeneity: An Experimental Approach in the Health and Retirement Study, Quarterly Journal of Economics, 112(2), 537-579

Boyle, G. \& Conniffe, D. (2008) Compatibility of expected utility and $\mu / \sigma$ approaches to risk for a class of non location-scale distributions. Economic Theory, 35, 343-336.

Clare, A. D., O’Brien, R., Thomas, S. H. \& Wickens, M. R. (1998) Macroeconomic shocks and the domestic CAPM: evidence from the UK Stock Market, International Journal of Finance and Economics. 3, 111-126

Ding, X., J.Hartog and Y.Sun (2010) Can we Measure Individuals Risk Attitudes in a Survey, Tinbergen Institute Discussion Paper, TI2010-027/03

Donkers, B., B. Melenberg, and A. V. Soest (2001), Estimating Risk Attitudes Using Lotteries: A Large Sample Approach, Journal of Risk and Uncertainty, 22(2), 165-195.

Engel, R. \& Rodrigues. A. P. (1989) Tests of international CAPM with time-varying Covariates. Journal of Applied Econometrics. 4, 119-138.

Feldstein, M. S. (1969) Mean-variance analysis in the theory of liquidity preference and portfolio selection. The Review of Economic Studies. 36, 5-12.

Flavin, T.J. (2006) How risk averse are fund managers? Evidence from Irish mutual Funds. Applied Financial Economics. 16, 1355-1363. 
Frankel, J (1985) “Portfolio Crowding Our Empirically Estimated,” Quarterly Journal of Economics, Vo. 100, pp. 1041-1065.

Friend, I, \& Blume, M. E. (1975) The demand for risky assets, American Economic Review. 65, 900-922

Giovanni, A. \& Jorion.P. (1989) The time variation of risk and return in the foreign exchange and stock markets. Journal of Finance, 44. 307-325

Guiso, L., and M. Paiella (2008), Risk Aversion, Wealth, and Background Risk, Journal of the European Economic Association, 6(6), 1109-1150,

Hartog, J., A. Ferrer-i-Carbonell and N. Jonker (2002), Linking measured risk aversion to individual characteristics, Kyklos, 55 (1), pp. 3-26

Hawawini, G (1978) “A Mean-Standard Deviation Exposition of the Theory of the Firm under Uncertainty: A Pedagogical Note,” American Economic Review, pp. 194-202.

Jianakoplos, N. (2002) “Invest As I Say, Not As I Do? Gender Differences In Financial Risk Preferences,” International Business \& Economics Research Journal, Vol. 1, no. 1, $1-8$.

Kocherlakota, N (1990), “On Tests of Representative Consumer Asset Pricing Models,” Journal of Monetary Economics, 26, pp. 285-304.

Levy H. \& Markowitz, H.H. (1979) Approximating expected utility by a function of mean and variance, American Economic Review. 69, 308-317.

Meyer, J. (1987) Two-Moment Decision Models and Expected Utility Maximisation. American Economic Review. 77, 421-430.

Meyer, D. J. \& Meyer, J. (2005) Relative risk aversion: What do we know? Journal of Risk and Uncertainty. 31, 243-262.

Rotthoff, K. (2011), "How Market Makers Affect Efficiency; Evidence Markets are Becoming Less Efficient,” Capital Markets Review, Vol. 18, Nos. 1\&2, 53-72.

Salem, A and T. Mount (1974) A Convenient Descriptive Model of Income Distribution: The Gamma Density, Econometrica, Vol. 42, no. 6, pp. 1115-1127.

Samuelson, P. A. (1970) The fundemental approximation theorem of portfolio analysis in terms of means, variances and higher moments. The Review of Economic Studies, 37, 537-542. 
Sinn, H.W. (1989). Economic Decisions under Uncertainty (Second English ed.) Amsterdam: North-Holland.

Thomas, S. \& Wickens M. R. (1993), An international CAPM for bonds and equities, Journal of International Money and Finance, 12, 390-412. 